\title{
Maternal quality of life in routine labor epidural analgesia versus labor analgesia on request: results of a randomized trial
}

\author{
A. A. S. van den Bosch ${ }^{1}$ (1) M. Goossens ${ }^{2} \cdot$ K. Bonouvrié ${ }^{3} \cdot$ B. Winkens ${ }^{4} \cdot$ J. G. Nijhuis ${ }^{1}$ · F. J. M. E. Roumen ${ }^{5}$ \\ M. M. L. H. Wassen ${ }^{6}$
}

Accepted: 19 March 2018 / Published online: 30 March 2018

(C) The Author(s) 2018

\begin{abstract}
Introduction The purpose of this study was to evaluate the changes in maternal quality of life (QOL) from pregnancy to 6 weeks after delivery between routine labor epidural analgesia (EA) and pain relief on maternal request only.

Methods IWomen delivering of a singleton in cephalic presentation beyond 36+0 weeks' gestation were randomly allocated to EA as a routine during labor (routine EA group), or to any kind of analgesia on request only (control group). The Short Form health survey (SF-36) was used to assess women's QOL before randomization, and 6 weeks postpartum. Data were analyzed according to the intention to treat principle.

Results A total of 488 women were included, and antepartum as well as postpartum SF-36 questionnaires were filled in by $356(73.0 \%)$ women, $176(49.4 \%)$ in the routine EA group, and $180(50.6 \%)$ in the control group. Changes from the QOL antepartum to the QOL 6 weeks postpartum were comparable between both groups, also in the subgroup of women in the control group who gave birth without any pain medication $(n=41,22.8 \%)$. Maternal age and the incidence of adverse events related to EA, which were both higher in the routine EA group, had no influence on the changes in QOL. Differences in request for pain relief were comparable with other studies.

Conclusion Routine administration of EA during labor and pain relief on maternal request only are associated with comparable changes of women's QOL antepartum to 6 weeks postpartum.
\end{abstract}

Keywords Analgesia $\cdot$ Childbirth $\cdot$ Epidural $\cdot$ Labor $\cdot$ Quality of life $\cdot$ Randomized trial

M. M. L. H. Wassen

m.wassen@zuyderland.nl

1 Department of Obstetrics and Gynecology, GROW School for Oncology and Developmental Biology, Maastricht University Medical Center+, PO Box 5800, 6202 Maastricht, AZ, The Netherlands

2 Department of Rehabilitation Research \& Clinical Psychological Sciences, Maastricht University, PO Box 616, 6200 Maastricht, MD, The Netherlands

3 The Department of Pediatrics, Antwerp University Hospital, Wilrijkstraat 10, 2650 Edegem, Belgium

4 Department of Methodology and Statistics, Maastricht University, PO Box 616, 6200 Maastricht, MD, The Netherlands

5 Department of Obstetrics and Gynecology, Zuyderland Medical Center Heerlen-Sittard, PO Box 4446, 6401 Heerlen, CX, The Netherlands

6 Department of Obstetrics and Gynecology, Zuyderland Medical Center Heerlen-Sittard, PO box 5500, 6130 Sittard, MB, The Netherlands

\section{Introduction}

Quality of life (QOL) is a multidimensional process that involves physical as well as mental aspects of life, influenced by subjective positive and negative evaluations [1-3]. Healthrelated quality of life has become a valid indicator of a population's well-being. It is a measure to assess the effects of treatment on physical, mental, emotional, and social functioning. Routine QOL questions about perceived physical and mental health and functioning became an important component of health surveillance, and are generally considered valid indicators of service needs as well as intervention outcomes [3]. Childbirth is an important critical aspect of life that can influence a women's QOL. It has been shown that women's subjective evaluation of childbirth has a major impact on maternal QOL after delivery $[4,5]$. Women with an uncomplicated pregnancy and childbirth have the highest QOL scores compared to women who have a complicated pregnancy and/or delivery [6]. Women with a negative childbirth experience 
have a harder time bonding with their child postpartum [7]. A negative childbirth experience may even result in a posttraumatic stress disorder, occurring in $2-6 \%$ of all deliveries [8-10].

Women's childbirth experience in turn is influenced by many factors, including socio-economic and demographic characteristics, pregnancy complications, labor characteristics, mode of delivery, neonatal outcome, and social support [5, 11-13]. One of these factors is the experience of labor pain [14]. Labor pain experience is subjective and multifactorial. Not having had a choice in pain relief, and not being satisfied in coping with pain during labor were associated with a negative birth experience [15]. Labor pain incidentally results in posttraumatic stress symptoms [9] or depressive symptoms, even more so when it results in persistent postpartum pain [16]. For many pregnant women, the fear of labor pain is also of major concern, resulting in severe fear of childbirth in 6-10\% [17]. This fear of pain can even result in a request for an elective cesarean section [18].

As the experience of labor pain contributes to the childbirth experience of women, optimal pain relief is an important issue. Maternal QOL during and after childbirth may be improved, if the health care professionals discuss labor pain and its management with the pregnant woman antepartum [19]. Epidural analgesia (EA) has been shown to be the most effective method of pharmacological pain relief during labor, as compared with other methods of labor analgesia [20-22]. A reduced risk of postpartum depression was demonstrated in women who received epidural analgesia, compared to no analgesia [23]. Non-inferiority of routine EA with regard to the risk of operative delivery could not be demonstrated in this trial. No important differences in labor and neonatal outcomes were found between both groups. However, routine EA use was likely to lead to more operative deliveries and more maternal adverse effects, including hypotension and motor blockade [24].

Because EA seems to affect not only a women's delivery but also her puerperium, we were interested to find out whether there would be a difference in QOL postpartum, in routine labor EA as compared with analgesia on request. We hypothesized that women who receive routine EA will have a greater change in QOL postpartum, as they might have a more positive childbirth experience because of EA during labor. The purpose of this study, therefore, was to evaluate the changes in QOL of women who received routine labor EA, as compared to women who were given pain relief on request only (control group), with special attention paid to the women who requested no pain relief at all.

\section{Methods}

\section{Design and patients}

This QOL study was linked to the recently published TREATtrial [24]. The trial was approved by the Medical Ethics Committee of the participating centres. It was registered in the clinical trial register (NCT01261689). The TREAT-trial was a bi-center, randomized, non-inferiority study that compared the effect of routine labor EA with a control group on the operative delivery rates, labor characteristics, maternal, and neonatal outcomes. Eligible women $(n=488)$ were included from September 2008 until May 2012 in one university and one non-university hospital in The Netherlands, thereby representing the labor care in different hospitals of the country. Women were considered to be eligible if they were 18 years or older and pregnant with a singleton in vertex presentation at a gestational age of 36 weeks or more, with the intention to deliver vaginally and without a contra-indication for EA. Women were asked to fill in the SF-36 antepartum before randomization (AP SF-36) at the outpatient clinic, and 6 weeks postpartum (PP SF-36) at their routine postpartum appointment. In case the PP SF-36 questionnaire was not returned, a research nurse tried to contact the women by telephone and asked them again to return the questionnaire.

\section{Randomization}

From 32 weeks' gestation, eligible women were given oral and written information about the TREAT-trial. After their oral and written informed consent, women were randomly allocated before the start (or induction) of labor to either routine EA $(n=233)$ or analgesia on request $(n=255)$ in a 1:1 ratio, with stratification for center and parity, by using sequentially numbered opaque envelopes. Additional information about the procedure is described in the original article [24].

\section{Intervention}

Women in the routine EA group received EA as soon as they were declared to be in labor, which was defined as an effaced cervix with at least 2 -cm dilation. Women in the control group received analgesia only on request, which could be opiates intramuscularly or EA, depending on the local hospital protocol (cervical dilation, progression of labor, expected time until full dilation) and the patient's preference.

\section{Health-related quality of life}

Health-related quality of life was a secondary outcome in the TREAT-trial; therefore, the sample size was not calculated specifically for this outcome. To evaluate the QOL, we used 
the Short Form 36 (SF-36). The Short Form 36 (SF-36) is a standardized generic health-related QOL questionnaire, which fulfills strict criteria of reliability and validity and is proven to be appropriate for the evaluation of QOL after delivery [12, 25-27]. The SF-36 consists of 36 items, and is subdivided in eight subscales: physical functioning, social functioning, role limitations because of physical health problems, roll limitations because of emotional problems, emotional well-being, vitality, bodily pain, and general health perception. A ninth separate subscale is general health change. Each subscale is ranged from 0 to 100 , where the higher the score the better the QOL [25].

\section{Statistical analysis}

Transformed scores of the SF-36 were calculated according to the SF-36 guideline as well as imputation of missing values [25]. If less than half of the items of a subscale were missing, then the missing values were determined by calculating the mean of the existing values [25].

Numerical variables were presented by mean $( \pm$ standard deviation) and categorical variables by number (\%). Independent-samples $t$ test and Chi-square or Fisher's exact test were performed to assess the differences in numerical and categorical variables between groups (EA vs. non-EA, analgesia on request vs. no analgesia on request), respectively.
Linear mixed effects models were used to analyze the difference in SF-36 scores between groups, since this method corrects for differences in AP scores, deals with the correlation between repeated measures within the same woman (AP and PP), and assumes missing at random (MAR), where a likelihood-based approach was used for missing outcomes. Moreover, we corrected for variables related to absence and/or to the outcome such as age, BMI, parity, complications during labor, and neonatal admittance [28]. A $p$ value $\leq 0.05$ was considered to be statistically significant. Statistical analyses were performed using IBM SPSS ${ }^{\circ}$ Statistics for Windows (version 23.0, IBM corp., Armonk, NY, USA).

\section{Results}

\section{Patient characteristics}

The QOL study population consisted of all women $(n=488)$ included in the TREAT trial (Fig. 1). From them, 233 $(47.7 \%)$ were allocated to the routine EA group and 255 $(52.3 \%)$ to the control group. In the routine EA group, 208 (89.3\%) women did receive EA and 25 (10.7\%) did not. Reasons for failure to get EA in this group were fast progression of labor $(n=15)$, technical problems $(n=2)$, unplanned cesarean section $(n=6)$, unavailability of EA $(n=1)$, and
Fig. 1 Flowchart of women participating in the TREAT-trial and included in the QOL analysis. Missing subscales or values of the SF-36 questionnaire were imputed or determined by calculating the mean of the existing values. $E A$ epidural analgesia

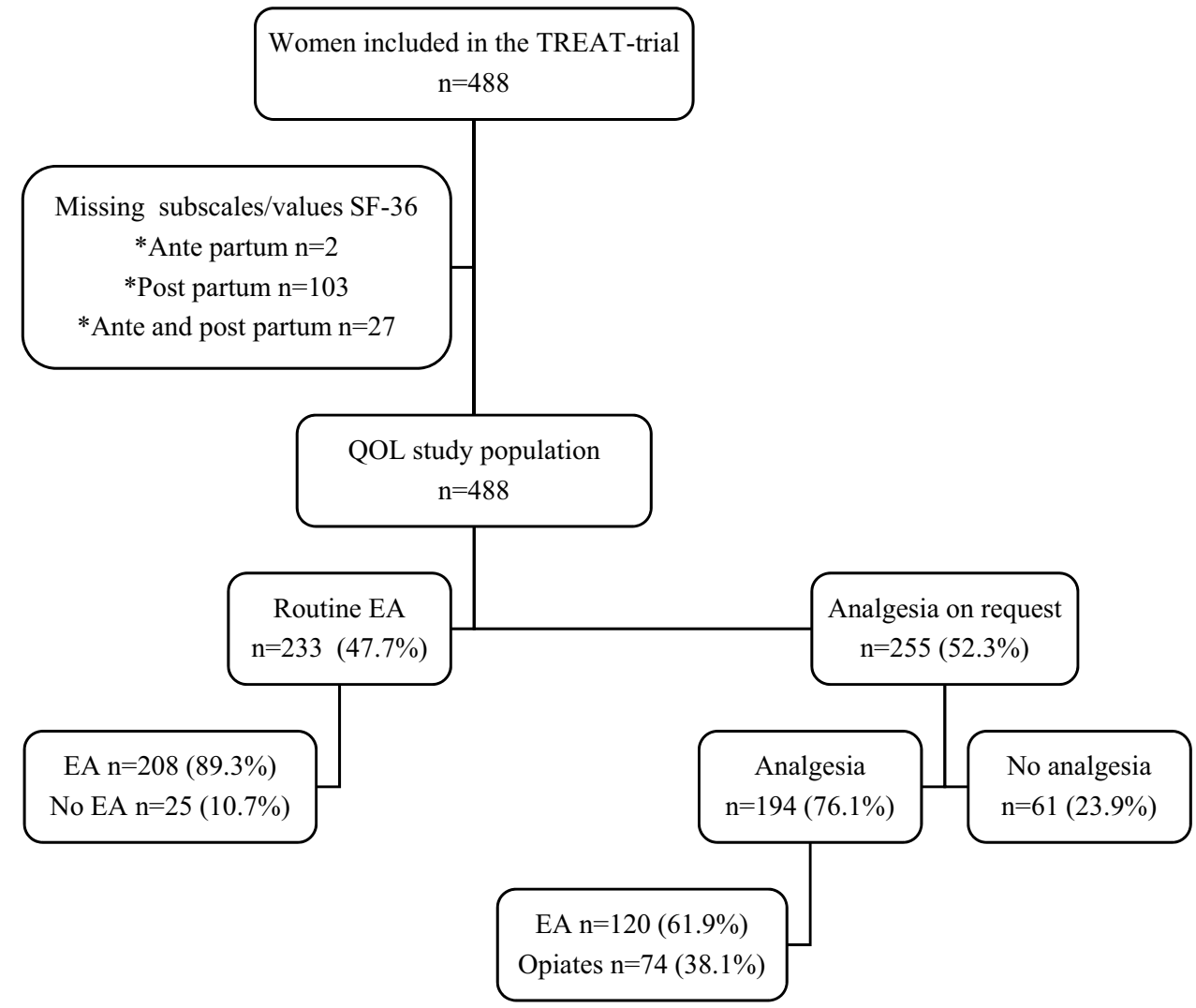


unknown $(n=1)$. From the 255 women who were allocated to the control group, 61 (23.9\%) gave birth without any pain medication. From the 194 women $(76.1 \%)$ who requested analgesia, 61 (30.9\%) received EA at first request, $59(30.4 \%)$ received EA at second request, and 74 women $(38.1 \%)$ received opiates. There were no significant differences between maternal baseline and labor characteristics between both groups (table not shown), except for a higher maternal age in the EA group $(p=<0.01)$ and more adverse events related to EA $(p=<0.00)$ in this group such as maternal hypotension and motor blockade.

The baseline characteristics of the women who did not fill out the AP SF-36 and/or PP SF-36 were not significantly different between the routine labor EA group and the control group (24.5 and $29.4 \%$, respectively; $p=0.26$; data not shown).

In the control group, women who delivered without analgesia $(n=61,23.9 \%)$, were older $(p \leq 0.00)$, had a lower BMI $(p \leq 0.00)$, were more often multiparous $(p \leq 0.00)$, received less frequently secondary education, even though primary and tertiary education levels were comparable. $(p=0.03)$, had more often a spontaneous vaginal delivery $(p \leq 0.01)$, and underwent less frequently an unplanned cesarean section $(p<0.04)$ as compared to the women who did receive pain medication $(n=194,76.1 \%)$.

\section{Changes in quality of life}

Table 1 shows the antepartum and postpartum scores on the nine subscales of the SF-36 for the routine EA group and the control group, as well as the difference in score changes between both groups. In both groups, QOL increased in six domains between antepartum and postpartum, but decreased for the domains 'role limitations due to emotional problems,' 'general health perception,' and 'general health change.' For 'physical functioning' and 'role limitations due to physical problems' the increase within both groups was even more than $20 \%$. However, there were no significant differences in score changes between the routine EA and the control group.

For the subgroups of the analgesia on request group, the antepartum and postpartum scores on the nine subscales of the SF-36 and the difference in change scores per subscale are shown in Table 2. There were no significant differences in the change scores between the subgroup of women who received analgesia on request and those who delivered without any analgesia.

\section{Discussion}

In this study, the maternal QOL was evaluated and compared between women who received routine EA during labor, and those who were given pain relief on request only. Women in both groups showed comparable changes in QOL from antepartum to 6 weeks postpartum, which is in line with other studies $[1,12]$. Our hypothesis, that routine labor EA would result in a significant improvement of QOL after delivery as compared with analgesia on request, could not be confirmed.

We used the SF-36 in this study to measure quality of life. The SF-36 is a generic tool to measure a person's quality of life and to compare this with other people's QOL. It has been used internationally and in cost-effectiveness studies (which was de primary intent of the TREAT-study). During pregnancy, a difference in both the routine EA group as well as the control group was observed, when compared to a general non-pregnant population studies ad described by van der Zee [25]. When comparing the SF-36 scores of our pregnant population with this general population, pregnant women scored lower on 5 out of the 9 subscales: physical functioning, social functioning, role limitations due to physical problems, emotional well-being, and bodily pain. This is in line with previous studies that showed an overall reduced QOL in women during pregnancy and the postpartum period $[1,29]$. This seems to imply that routinely given pain relief does not influence women's health-related QOL.

As women participating in the TREAT trial were well informed about the possibilities of labor pain relief, this might have resulted in a decrease of fear of pain as well as pain experience during delivery in these women. In turn this may have had a positive effect on their birth experience.

In the control group, nearly $24 \%$ of women delivered without request for any kind of analgesia. Compared with women who received analgesia in the control group, there were a few statistical significant differences. Women who received analgesia in the control group were older, more often multiparous, higher educated and had a lower BMI. Also, mode of delivery was more often spontaneously and less had an unplanned cesarean section. The fact that multiparous women less often choose EA during labor can be explained by positive previous delivery experiences which may also play a role in increased confidence resulting in decreased fear of pain in multiparous women [30]. It is likely that women who choose not to request analgesia are more confident in their own ability to handle pain and their physical functioning than women who do not have this confidence. The higher maternal age in this group can probably be linked to this multiparity. Other studies also showed a positive relationship between a higher BMI $\left(>25 \mathrm{~kg} / \mathrm{m}^{2}\right)$ and request for EA analgesia, but showed a negative relationship between higher education and EA request [31, 32]. However, these differences in characteristics did not result in a significant change of QOL after delivery, as compared with the women who did receive analgesia on request. Nevertheless, we found a nearly significant difference in the dimension physical 
Table 1 Estimated means of transformed SF-36 subscale scores* and score change of the subscales, before randomization (AP) and 6 weeks postpartum (PP) in the QOL study population and in the control group

\begin{tabular}{|c|c|c|c|c|}
\hline & $\begin{array}{l}\text { Routine epidural analgesia } \\
(n=233)\end{array}$ & $\begin{array}{l}\text { Control group } \\
(n=255)\end{array}$ & Score change $(95 \% \mathrm{CI})$ & $p$ Value \\
\hline Physical functioning & & & $1.5(-3.53-6.49)$ & 0.561 \\
\hline $\mathrm{AP} *$ & $66.2 \pm 2.0$ & $66.8 \pm 1.9$ & & \\
\hline $\mathrm{PP}^{* *}$ & $88.3 \pm 1.8$ & $87.4 \pm 1.8$ & & \\
\hline Social functioning & & & $0.38(-4.76-5.52)$ & 0.883 \\
\hline AP & $75.5 \pm 2.0$ & $73.7 \pm 1.9$ & & \\
\hline PP & $79.7 \pm 2.1$ & $77.6 \pm 2.2$ & & \\
\hline Role limitation due to physical problems & & & $-3.59(-13.49-6.31)$ & 0.476 \\
\hline $\mathrm{AP}$ & $39.0 \pm 3.4$ & $37.1 \pm 3.3$ & & \\
\hline PP & $63.3 \pm 3.5$ & $65.0 \pm 3.6$ & & \\
\hline Role limitation due to emotional problems & & & $-1.79(-8.86-5.27)$ & 0.618 \\
\hline AP & $88.4 \pm 2.4$ & $86.9 \pm 2.4$ & & \\
\hline PP & $83.4 \pm 2.9$ & $83.7 \pm 2.9$ & & \\
\hline Emotional well-being & & & $1.24(-1.79-4.28)$ & \\
\hline $\mathrm{AP}$ & $72.3 \pm 1.3$ & $72.9 \pm 1.3$ & & \\
\hline PP & $80.1 \pm 1.4$ & $79.5 \pm 1.4$ & & 0.420 \\
\hline Vitality & & & $0.22(-3.50-3.95)$ & 0.906 \\
\hline $\mathrm{AP}$ & $55.1 \pm 1.5$ & $54.2 \pm 1.5$ & & \\
\hline PP & $58.2 \pm 1.7$ & $57.1 \pm 1.7$ & & \\
\hline Bodily pain & & & $-0.55(-6.07-4.98)$ & 0.846 \\
\hline $\mathrm{AP}$ & $66.4 \pm 2.0$ & $64.1 \pm 2.0$ & & \\
\hline PP & $74.7 \pm 2.1$ & $73.0 \pm 2.1$ & & \\
\hline General health perception & & & $0.54(-2.22-3.30)$ & 0.702 \\
\hline $\mathrm{AP}$ & $78.0 \pm 1.5$ & $75.4 \pm 1.4$ & & \\
\hline PP & $77.5 \pm 1.6$ & $74.3 \pm 1.5$ & & \\
\hline General health change & & & $0.65(-3.38-4.67)$ & 0.753 \\
\hline $\mathrm{AP}$ & $50.9 \pm 1.5$ & $52.5 \pm 1.5$ & & \\
\hline PP & $49.8 \pm 1.6$ & $50.7 \pm 1.6$ & & \\
\hline
\end{tabular}

$p$ value $\leq 0.05$ was considered to be statistically significant

$A P$ Antepartum, $P P$ Postpartum

*Range from 0 to 100

functioning in favor of the women who delivered without any pain medication $(p=0.054)$. Significance could presumably have been demonstrated if the number of women who delivered without any pain medication (now, $n=61$ ) had been comparable in size with the number of women who received pain medication on request (now, $n=194$ ).

The strength of this study is that it is the first randomized controlled trial, evaluating the effect of routine labor EA on maternal QOL, 6 weeks postpartum. The SF-36 questionnaire used in this study is a standard and well-known non-specific health-related QOL questionnaire, which has been validated for the evaluation of QOL after delivery [12, 13, 27, 33].

The study also has some limitations. Firstly, we cannot exclude selection bias of the women participating in the TREAT-trial, and thus also in the QOL study, as it is likely that these women favored EA for labor pain treatment more than those women who refused participation.

Secondly, our follow-up period of 6 weeks is relatively short. Not all women will have recovered completely in 6 weeks. Delivery-related problems like urinary incontinence, perineal pain, and chronic exhaustion may have an effect on the QOL when these women return to their daily activities after their maternity period is completed [34]. However, the mode of delivery (spontaneous, vaginal instrumental or unplanned cesarean section) would only have a small impact on these results, as these labor characteristics were not significantly different between both groups. Besides, during the routine postpartum check-up at 6 weeks, no women reported adverse effects of EA such as hypotension or motor blockade. Postpartum depression is most common between 6 and 12 weeks postpartum, and can have a 
Table 2 Estimated means of transformed SF-36 subscale scores* and score change of the subscales, before randomization (AP) and 6 weeks postpartum (PP) in the control group - analgesia on request versus no analgesia on request

\begin{tabular}{|c|c|c|c|c|}
\hline & $\begin{array}{l}\text { Analgesia on request } \\
(n=194)\end{array}$ & $\begin{array}{l}\text { No analgesia on request } \\
(n=61)\end{array}$ & Score change $(95 \% \mathrm{CI})$ & $p$ Value \\
\hline Physical functioning & & & $7.94(-0.12-16.00)$ & 0.054 \\
\hline $\mathrm{AP} *$ & $67.9 \pm 2.3$ & $67.2 \pm 3.7$ & & \\
\hline $\mathrm{PP}^{* *}$ & $86.7 \pm 2.2$ & $93.9 \pm 3.3$ & & \\
\hline Social functioning & & & $-0.51(-9.50-8.48)$ & 0.911 \\
\hline AP & $73.0 \pm 2.4$ & $76.7 \pm 3.7$ & & \\
\hline PP & $76.9 \pm 2.8$ & $80.1 \pm 4.3$ & & \\
\hline Role limitation due to physical problems & & & $4.50(-12.22-21.22)$ & 0.596 \\
\hline $\mathrm{AP}$ & $39.3 \pm 4.0$ & $37.6 \pm 6.3$ & & \\
\hline $\mathrm{PP}$ & $66.1 \pm 4.5$ & $69.0 \pm 7.0$ & & \\
\hline Role limitation due to emotional problems & & & $-0.08(-11.56-11.40)$ & 0.989 \\
\hline $\mathrm{AP}$ & $83.9 \pm 3.1$ & $88.2 \pm 4.7$ & & \\
\hline PP & $80.6 \pm 3.5$ & $84.9 \pm 5.5$ & & \\
\hline Emotional well-being & & & $-2.41(-7.17-2.34)$ & 0.318 \\
\hline $\mathrm{AP}$ & $73.0 \pm 1.6$ & $74.3 \pm 2.5$ & & \\
\hline PP & $80.1 \pm 1.8$ & $79.0 \pm 2.9$ & & \\
\hline Vitality & & & $-4.53(-11.05-1.98)$ & 0.172 \\
\hline $\mathrm{AP}$ & $54.5 \pm 1.9$ & $57.0 \pm 2.8$ & & \\
\hline PP & $58.4 \pm 2.1$ & $56.4 \pm 3.3$ & & \\
\hline Bodily pain & & & $3.32(-5.90-12.53)$ & 0.479 \\
\hline $\mathrm{AP}$ & $64.9 \pm 2.4$ & $65.8 \pm 3.8$ & & \\
\hline PP & $72.9 \pm 2.7$ & $77.1 \pm 4.3$ & & \\
\hline General health perception & & & $2.28(-2.63-7.20)$ & 0.361 \\
\hline $\mathrm{AP}$ & $76.1 \pm 1.9$ & $75.1 \pm 2.8$ & & \\
\hline PP & $74.5 \pm 2.0$ & $75.7 \pm 3.1$ & & \\
\hline General health change & & & $2.70(-4.02-9.44)$ & 0.428 \\
\hline $\mathrm{AP}$ & $53.3 \pm 1.9$ & $51.5 \pm 3.0$ & & \\
\hline PP & $50.8 \pm 2.1$ & $51.8 \pm 3.3$ & & \\
\hline
\end{tabular}

$p$ value $\leq 0.05$ was considered to be statistically significant

AP Antepartum, PP Postpartum

*Range from 0 to 100

major effect on the maternal QOL [34, 35]. Previous studies compared the QOL at 6 weeks and 6 months postpartum [34, 36, 37], and even at 2 years after delivery [16]. They evaluated the effect of earlier induction on QOL $[36,37]$ or the relationship between physical and emotional health problems [34]. Even though these studies did not have the same intervention, they showed higher QOL scores 6 months postpartum compared to 6 weeks. Evaluation at longer periods after childbirth might give a better insight into the long-term effect of pregnancy and labor on women's QOL.

Thirdly, the TREAT-trial did not have quality of life as a primary outcome. The study was powered to show noninferiority of routine use of epidural analgesia, and therefore, it is possible that this study was underpowered to show a statistical significant difference in QOL between the two groups. We conclude that the routine administration of
EA during labor and pain relief on request are associated with comparable changes of women's QOL antepartum to 6 weeks postpartum, whereas women in the routine EA group had more adverse events related to EA. Based on the changes in women's QOL, no preference can be given to routine labor EA, as compared with labor analgesia on request. Future studies may focus on the long-term effect of routine EA on the postpartum QOL.

\section{Compliance with ethical standards}

Conflict of interest The authors report no conflict of interest.

Open Access This article is distributed under the terms of the Creative Commons Attribution 4.0 International License (http://creativeco mmons.org/licenses/by/4.0/), which permits unrestricted use, distribution, and reproduction in any medium, provided you give appropriate 
credit to the original author(s) and the source, provide a link to the Creative Commons license, and indicate if changes were made.

\section{References}

1. Otchet, F., Carey, M. S., \& Adam, L. (1999). General health and psychological symptom status in pregnancy and the puerperium: What is normal? Obstetrics \& Gynecology, 94(6), 935-941.

2. The WHOQOL GRoup. (1998). The World Health Organization Quality of Life Assessment (WHOQOL): Development and general psychometric properties. Social Science \& Medicine, 46, 1569-1585.

3. Hennessy, C. H., et al. (1994). Measuring health-related quality of life for public health surveillance. Public Health Reports, 109(5), $665-672$.

4. Gerard Jansen, A. J., Wim, J. J. D., \& Hop, C. J. (2007). MarieLouise Essink-Bot, New insights into fatigue and health-related quality of life after delivery. Acta Obstetricia et Gynecologica, 86, 579-584.

5. Mousavi, S. A., et al. (2013). Quality of life after cesarean and vaginal delivery. Oman Medical Journal, 28(4), 245-251.

6. Waterstone, M., et al. (2003). Postnatal morbidity after childbirth and severe obstetric morbidity. BJOG, 110(2), 128-133.

7. Simpson, M., \& Catling, C. (2015). Understanding psychological traumatic birth experiences: A literature review. Women and Birth, 29(3), 203-207.

8. Susan, A., et al. (2009). Posttraumatic stress disorder after childbirth: Analysis of symptom presentation and sampling. Journal of Affective Disorders, 119(1-3), 200-204.

9. Garthus-Niegel, S., et al. (2014). The role of labor pain and overall birth experience in the development of posttraumatic stress symptoms: A longitudinal cohort study. Birth, 41(1), 108-115.

10. Wijma, K., Soderquist, J., \& Wijma, B. (1997). Posttraumatic stress disorder after childbirth: A cross sectional study. Journal of Anxiety Disorders, 11(6), 587-597.

11. Kavosi, Z., et al. (2015). A comparison of mothers' quality of life after normal vaginal, cesarean, and water birth deliveries. International Journal of Community Based Nursing and Midwifery, 3(3), 198-204.

12. Jansen, A. J., et al. (2007). Psychometric evaluation of healthrelated quality of life measures in women after different types of delivery. Journal of Psychosomatic Research, 63(3), 275-281.

13. Torkan, B., et al. (2009). Postnatal quality of life in women after normal vaginal delivery and caesarean section. BMC Pregnancy Childbirth, 9, 4.

14. Melzack, R. (1993). Labour pain as a model of acute pain. Pain, 53(2), 117-120

15. Rijnders, M., et al. (2008). Perinatal factors related to negative or positive recall of birth experience in women 3 years postpartum in the Netherlands. Birth, 35(2), 107-116.

16. Bijl, R. C., et al. (2016). A retrospective study on persistent pain after childbirth in the Netherlands. Journal of Pain Research, 9, $1-8$

17. Flink, I. K., et al. (2009). Pain in childbirth and postpartum recovery: The role of catastrophizing. European Journal of Pain, 13(3), 312-316.

18. Dehghani, M., Sharpe, L., \& Khatibi, A. (2014). Catastrophizing mediates the relationship between fear of pain and preference for elective caesarean section. European Journal of Pain, 18(4), 582-589.
19. Carvalho, B., Zheng, M., \& Aiono-Le, L., \& Tagaloa (2014). A prospective observational study evaluating the ability of prelabor psychological tests to predict labor pain, epidural analgesic consumption, and maternal satisfaction. Anesthesia and Analgesia, 119(3), 632-640.

20. Anim-Somuah, M., Smyth, R. M., \& Jones, L. (2011). Epidural versus non-epidural or no analgesia in labour. Cochrane Database of Systematic Reviews, (12). https://doi.org/10.1002/14651 858.CD000331.pub3

21. NVA en NVOG. (2008). Richtlijn medicamenteuze pijnbehandeling tijdens de bevalling. Utrecht: NVA en NVOG

22. Freeman, L. M., et al. (2015). Patient controlled analgesia with remifentanil versus epidural analgesia in labour: Randomised multicentre equivalence trial. $B M J, 350, \mathrm{~h} 846$.

23. Hiltunen, P., et al. (2004). Does pain relief during delivery decrease the risk of postnatal depression? Acta Obstetricia et Gynecologica Scandinavica, 83(3), 257-261.

24. Wassen, M. M., et al. (2015). Routine labour epidural analgesia versus labour analgesia on request: A randomised non-inferiority trial. BJOG, 122(3), 344-350.

25. van der Zee, K. I., Sanderman, R. (2012). Het meten van de algemene gezondheidstoestand met de RAND-36. Groningen: NCG.

26. Brazier, J. E., et al. (1992). Validating the SF-36 health survey questionnaire: New outcome measure for primary care. $B M J$, 305(6846), 160-164

27. Hays, R. D., Sherbourne, C. D., \& Mazel, R. M. (1993). The RAND 36-Item Health Survey 1.0. Health Economics, 2(3), 217-227.

28. Mortazavi, F., et al. (2014). Maternal quality of life during the transition to motherhood. Iranian Red Crescent Medical Journal, 16(5), e8443.

29. Haas, J. S., et al. (2005). Changes in the health status of women during and after pregnancy. Journal of General Internal Medicine, 20(1), 45-51.

30. Lowe, N. K. (1989). Explaining the pain of active labor: The importance of maternal confidence. Research in Nursing \& Health, 12(4), 237-245.

31. Koteles, J., et al. (2012). Maternal characteristics and satisfaction associated with intrapartum epidural analgesia use in Canadian women. International Journal of Obstetric Anesthesia, 21(4), 317-323.

32. Orejuela, F. J., et al. (2012). Exploring factors influencing patient request for epidural analgesia on admission to labor and delivery in a predominantly Latino population. Journal of Immigrant and Minority Health, 14(2), 287-291.

33. Mogos, M. F., et al. (2013). A systematic review of quality of life measures in pregnant and postpartum mothers. Applied Research in Quality of Life, 8(2), 219-250.

34. Brown, S., \& Lumley, J. (2000). Physical health problems after childbirth and maternal depression at six to seven months postpartum. BJOG, 107(10), 1194-1201.

35. Sadat, Z., et al. (2014). The impact of postpartum depression on quality of life in women after child's birth. Iranian Red Crescent Medical Journal, 16(2), e14995.

36. Bijlenga, D., et al. (2011). Maternal health-related quality of life after induction of labor or expectant monitoring in pregnancy complicated by intrauterine growth retardation beyond 36 weeks. Quality of Life Research, 20(9), 1427-1436.

37. Bijlenga, D., et al. (2011). Health-related quality of life after induction of labor versus expectant monitoring in gestational hypertension or preeclampsia at term. Hypertension in Pregnancy, $30(3), 260-274$. 